\title{
Artificial Bee Colony Optimization Algorithm for Enhancing Routing in Wireless Networks
}

\author{
Dr. I. Jeena Jacob, \\ Department of Computer Science and Engineering, \\ GITAM University, Bangalore, India. \\ jeenajacob2016@gmail.com
}

\section{Dr. P. Ebby Darney,}

Professor, Department of EEE,

SCAD CET,

Tirunelveli, India.

darney.pebby@gmail.com

\begin{abstract}
The throughput of wireless multi-channel networks are enhanced using artificial intelligence algorithm. The performance of the network may be improved while reducing the interference. This technique involves three steps namely creation of wireless environment specific model, performance optimization using the right tools and improvement of routing by selecting the performance indicators cautiously. Artificial bee colony optimization algorithm and its evaluative features positively affects communication in wireless networks. The simple behavior of bee agents in this algorithm assist in making synchronous and decentralized routing decisions. The advantages of this algorithm is evident from the MATLAB simulations. The nature inspired routing algorithm offers improved performance when compared to the existing state-of-the-art models. The simple agent model can improve the performance values of the network. The breadth first search variant is utilized for discovery and deterministic evaluation of multiple-paths in the network increasing the overall routing protocol output.
\end{abstract}

Keywords: Wireless networks, throughput, routing, artificial intelligence, artificial bee colony

\section{Introduction}

Increasing utilization of portable and mobile devices has made wireless networks an essential component of communication [1]. The increasing network resource demands imposes the need for increasing the network capacity. Efficient algorithms and protocols are essential to increase the network capacity in a wireless environment. These algorithms also help in increasing the 
Journal of Artificial Intelligence and Capsule Networks (2021)

Vol.03/ No.01

Pages: $62-71$

http://irojournals.com/aicn/

DOI: https://doi.org/10.36548/jaicn.2021.1.006

wireless network throughput. Data reception and transmission is performed by multiple nodes in a wireless network [2]. Efficient data transfer to the network nodes are ensured by appropriate design of network protocols. The wireless network operation is largely dependent on these protocols. In order to support the functions of new generation networks, more efficient techniques and schemes are essential. Several researchers are working on developing state-ofthe art techniques to meet the needs of the modern networks. The natural and biological networks of organisms and insects and analyzed to design advanced and modern wireless networks [3]. The geographically distributed users are searched and connected using the wireless networks with a strong sense.

Over long distances, the wireless network signal strength varies due to the environmental impact and physical conditions. Reliable data transmission has to be ensured between the source and destination by wider transmission of signals across the area of sensor network. Along with other advantages, in wide network areas, wireless networks are beneficial [4]. The performance of the nodes can be observed from within the network using a cognitive system. The network elements self-recovery depends on the network capacity in this system [5]. Knowledge plane (KP) is the key element that may be used for incorporating system regulation, learning and thinking features in a distributed cognitive system. High-level version of network model may be created and maintained using this technique [6]. Further, the network throughput may be increased by ensuring efficient routing decision to be made in the network in any specific conditions. The knowledge based networks may be enhanced by enabling global and local routing of information and reasoning and other advanced features by means of knowledge plan. The network throughput can be enhanced while improving the routing efficiency using this knowledgeable feature [7]. Suitable instructions are fed to the cognitive system to identify the concept by a single person in previously introduced hypotheses. The node cognition characteristics are understood by the system for evaluation of network performance and control of other network elements. In order to obtain improved results, complete understanding of the network environment is essential [8].

Real time inference of the network and sensor network parts is essential. The sensor networks are the major elements of the network architecture [9]. All the network elements must be associated with each other if multiple units are present in the cognitive network. All the corresponding units must be associated when various network units are reconfigured in a repeated manner with multiple elements for solving cognitive issues. The cognitive networks 
Journal of Artificial Intelligence and Capsule Networks (2021)

Vol.03/ No.01

Pages: $62-71$

http://irojournals.com/aicn/

DOI: https://doi.org/10.36548/jaicn.2021.1.006

may de designed and evaluated using specifically designed architectures. Three layers, namely the datalink layer, physical and network layer has to collaborate with each other. Two propositions are available for this deliberation, one each for the lower and upper layer. Cross layer optimization is made possible with this technique [10]. Network optimization is performed by enabling end to end node operation of cognitive networks at each layer.

\section{Materials and Schemes}

Artificial intelligence ideas are used for enhancing the network throughput using flexible wireless routing algorithm. Data transfer over long distances between the source and destination is a major advantage of wireless networks [7]. Best path is chosen between the sender and receiver for increasing the data transmission speed in the network. The next shortest route is used for routing the data packets if the route is busy. However, certain drawbacks like interference exists in wireless networks. Same bandwidth and channel are shared by several nodes in the network in this scheme. The overall network throughput is affected due to interference that occurs between the transmitted data and the routes influencing the results. The impetus to design certain scenarios is the diverse parameters and their performance values varying in a systematic manner as well as the wide operation environment coverage range [11].

Brainstorming of the algorithm can be performed for covering the network engineering's three categories in wide range namely quantum traffic engineering, hybrid traffic engineering and real world applications. Repeatable abstraction patterns [12] are available when quantum traffic engineering is used for achieving improved statistical performance values over multiple attempts. Five experiments are conducted and their average is considered for estimation of the performance values. The algorithm based stochastic values are eliminated by the average values obtained. G.711 codec is used along with parallel Voice over IP sessions [13] and one sample per packet for handling the multimedia traffic with the proposed algorithm in diverse ways or in a similar manner. An approximate of $64 \mathrm{kbps}$ speed is required for accurate operation of the Voice over IP session. Identical packet delay and jitter values are delivered successfully along with other requirements using the proposed algorithm. Simulating and implementing similar ideas are a fascinating results of the current experimentation. The existing software and hardware resources are used for executing the realizable optimized artificial bee colony algorithm using core 2 duo processor in the current experiment. Utilization 
Journal of Artificial Intelligence and Capsule Networks (2021)

Vol.03/ No.01

Pages: $62-71$

http://irojournals.com/aicn/

DOI: https://doi.org/10.36548/jaicn.2021.1.006

of this algorithm in real world networks has provided several noticeable benefits. This algorithm may also be tested on large scale network layouts.

Extracted data is converted into real values with the help of data science. The data is learnt and predictions are made by several machine learning, deep learning and other artificial intelligence algorithms. In various wireless communication aspects, smart capabilities and context awareness is enabled by learning mechanisms in wireless network context. The quality of service and network-wide performance enhancement has led to the success and popularity of these algorithms over the years. In the wireless environments that are dynamically varying and complex in nature, these algorithms adapt themselves due to their intelligent behavior. Self-optimization and self-healing concepts are realized while adding automation facilities in wireless networks. In cellular networks, cognitive radio networks, wireless body area networks, wireless sensor networks, and mobile ad hoc network context, several data driven approaches are analyzed over the past few years. Spectrum sensing, energy harvesting communication, localization, data clustering and aggregation, routing, medium access control and various other topics are addressed using these approaches. Machine learning algorithms are used for solving issues related to anomaly detection, clustering, classification and regression.

\section{Results and Discussions}

The experimental results deduced are elaborated in this section. The simulation results and results obtained on implementing the algorithm on real time networks are discussed. A novel framework is designed for verification of the performance of the protocol. MATLAB is used for testing and implementation of the model. MATLAB results deduce that similar patterns and outcomes are seen on specified algorithms based on the traffic pattern results. Linux operating system is used for comparison of pattern generation on virtual machines using multiple real networks. The Artificial bee colony optimization network protocol enable easy tracing of similarity concepts in real time as well as virtual networks. Various real world networks are set up for experimentation purpose. The intelligent routing protocols are implemented and compared in these environments.

\subsection{Network Traffic Learning Experiments}

The traffic network throughput, its learning and enhancements are checked with the help of certain experiments. The values of various parameters are altered during the conduct of 
Journal of Artificial Intelligence and Capsule Networks (2021)

Vol.03/ No.01

Pages: $62-71$

http://irojournals.com/aicn/

DOI: https://doi.org/10.36548/jaicn.2021.1.006

the experiments. When the network is operating under saturated load, its behavior is to be determined. Each parameter is altered individually to understand the performance of the network. In network traffic load, its behavior is observed by varying the number of iterations along with the parameters. MATLAB software is used for executing these experiments with a real time network using Linux router. The virtual machine values and the parameter values are chosen to be identical to each other. It has been observed that the performance of the network is impacted in a positive manner with the increase in the number of iterations in these experiments. The output is increased by the smart routing protocol as it learns from the external operational structure. Figure 1, 2 and 3 provides the output of best cost with respect to the number of iterations of every simulation.

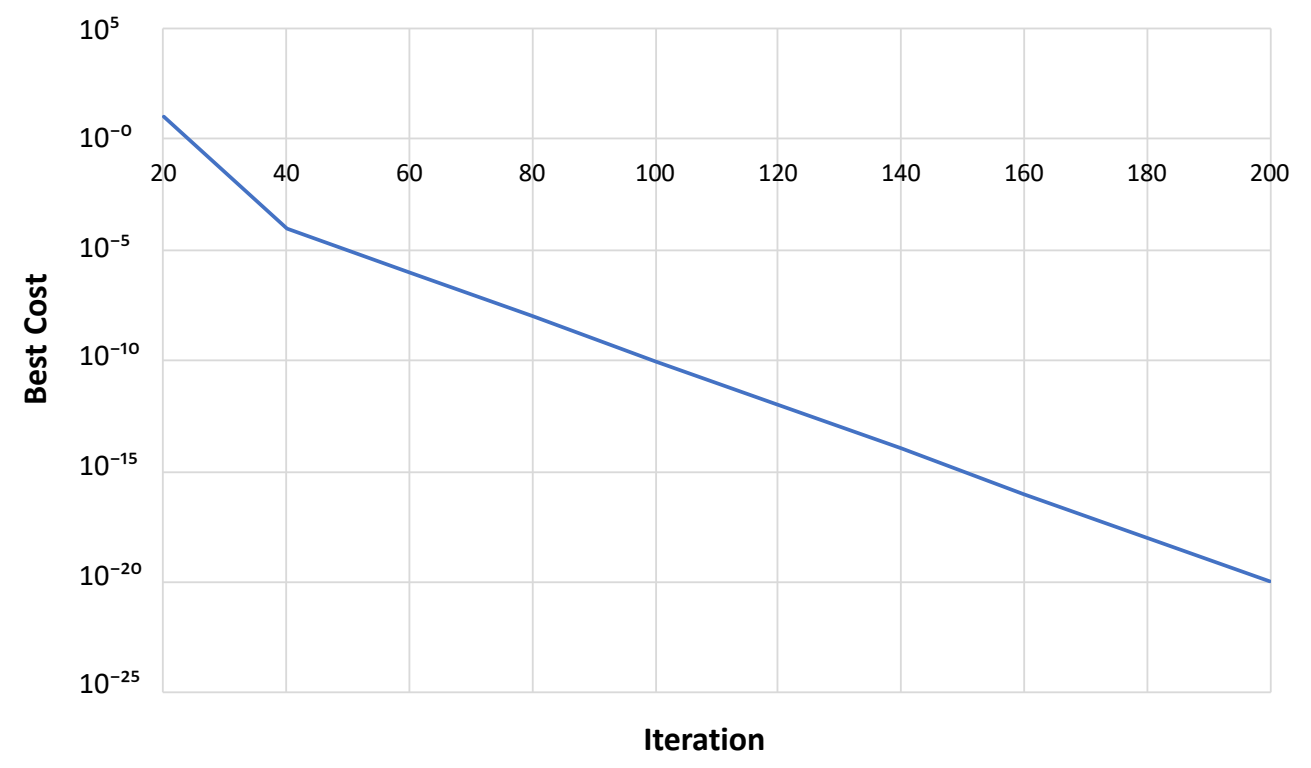

Figure 1: Simulation for 200 iterations

It is evident from the simulation results that, with the increase in the number of iterations, the number of packets delivered also increases. As time progresses, the routing algorithm learns from the wireless network thereby increasing the resulting output. Due to the queueing feature, certain packets are delayed in the network. However, the simulation sessions and real world networks show significant variation in this delay. Transmission mode control, forward error correction, coding, bit-by-bit delivery and modulation services are managed by the wireless physical layer while maintaining several features of the bits, signals and the 
Journal of Artificial Intelligence and Capsule Networks (2021)

Vol.03/ No.01

Pages: $62-71$

http://irojournals.com/aicn/

DOI: https://doi.org/10.36548/jaicn.2021.1.006

physical medium. For several applications, artificial neural networks (ANN) can be used for improving the performance of the wireless physical layer.

When the transmission channel model is unknown, end-to-end transmission can be modelled using feed forward neural network. This algorithm can help mapping the gain values of the channel to the hidden layers, receiver to the output layers and the transmitter to the input layers. This eliminates the need for exclusive mathematical model for the channel. The signal transmission quality can be improved by spiking neural networks (SNN) as signal correction and detection can be performed on the continuous data using this algorithm. The received signals can be detected by the learned patterns from the signals using the SSN. Detection and correction of signal errors may also be performed. Due to their ability of data extraction and storage, modulation classification can be performed using convolution neural networks (CNN) and deep neural networks (DNN). Channel decoding, learning transmitted symbols and signal detection in physical layer design and other such issues are studied with the help of ANNs. In applications like collision detection, carrier sensing, classification, modulation control, signal correction, signal detection, channel coding and decoding, physical layer design can be performed with the help of ANNs.

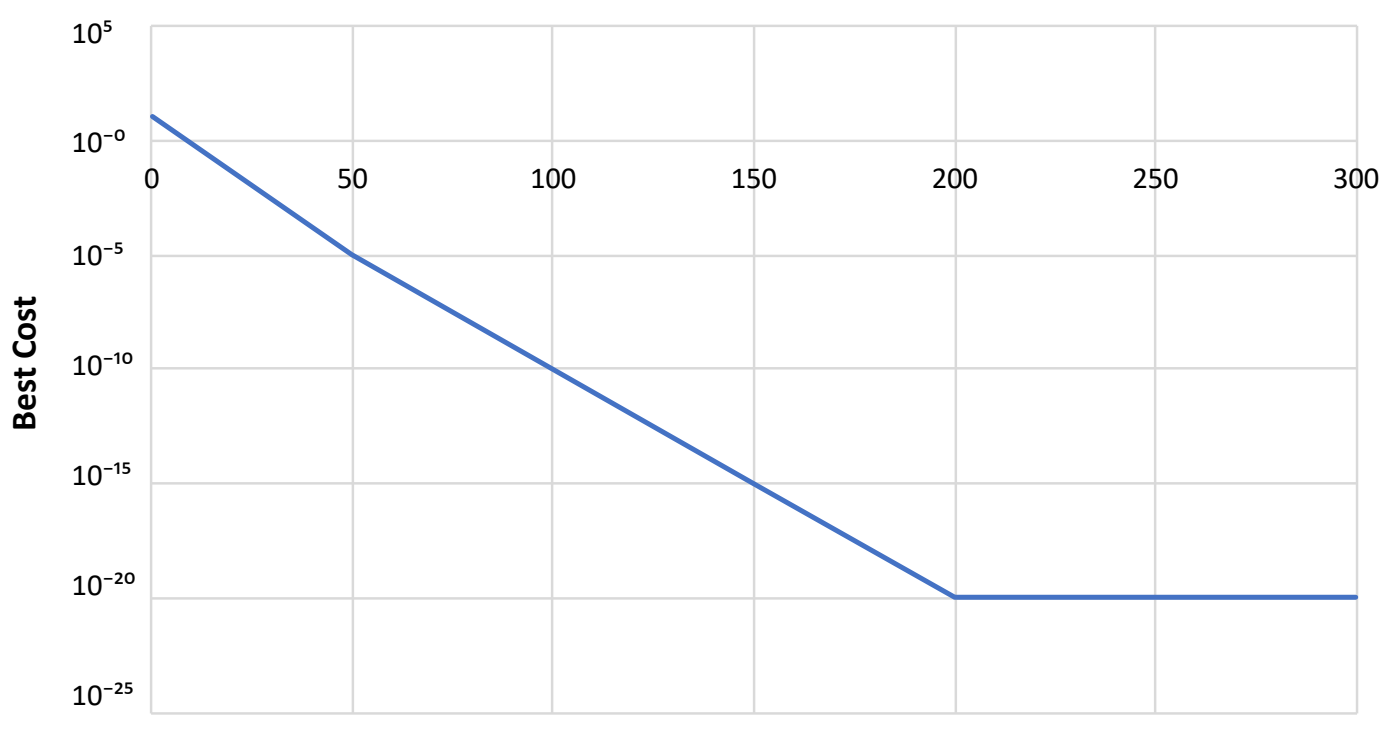

Iteration

Figure 2: Simulation for 300 iterations

There is a time varying weight value or connection strength among two neurons due to the time varying activation value of every spiking neuron in SNN. Depression or potentiation 
Journal of Artificial Intelligence and Capsule Networks (2021)

Vol.03/ No.01

Pages: $62-71$

http://irojournals.com/aicn/

DOI: https://doi.org/10.36548/jaicn.2021.1.006

is the term used for representing the weakening or weight strengthening in SNN. Based on the impact of change, it is categorized into short term and long term. Short-term depression (STD), short-term potentiation (STP), long term depression (LTD) and long term potentiation (LTP) are the major classifications based on the weight change impact duration. The precise timing of spikes present affects the adjustment of connection strength in the spiking neuron networks which is demonstrated commonly in neurobiological research.

Deep SNN is a remarkable DNN architecture. The energy efficiency and latency of DNN can be improved to a great extent using this architecture due to its event-based computational features. In real time applications where power consumption and speed factors are significant, deep SNNs may be used due to its advanced features. Despite its computational efficiency, there is an inadequacy of training algorithms for deep SNNs. The spike signals are not differentiable. Whenever the threshold condition is crossed by the internal state, the spiking neuron produces discrete events. However, when error backpropagation is used, differentiable activation functions are essential. Due to these reasons, training DNNs is done most commonly using this algorithm. In various domains, deep SNN is used in a sparse manner. However, these limitations may be overcome by integration of other cutting edge technologies.

Self-organization and other smart operations as well as prediction functions that are hugely independent from each other can be performed simultaneously using ANN. New data can be generated by decision making and decision making is enabled by data. ANN can be used for prediction of user behaviors like content requests and head movement in wireless networks for virtual reality applications. ANN-based RL algorithms and their predictions enable improvement of quality of service due to their spectral and computational resource allocation features. 
Journal of Artificial Intelligence and Capsule Networks (2021)

Vol.03/ No.01

Pages: $62-71$

http://irojournals.com/aicn/

DOI: https://doi.org/10.36548/jaicn.2021.1.006

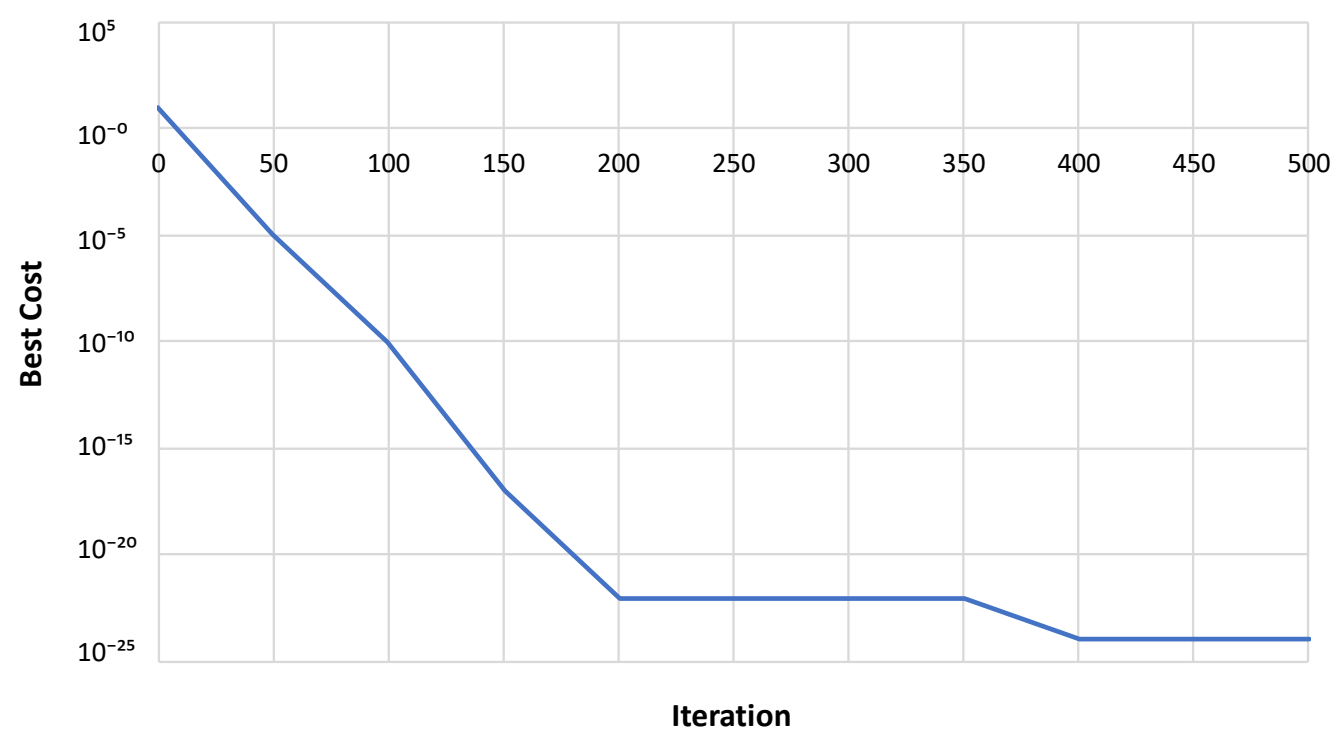

Figure 3: Simulation for 500 iterations

\section{Conclusion}

The technical and scientific contributions of this paper is emphasized in a comprehensive conclusion. Knowledgeable and intelligent routers are emphasized and natural engineering is incorporated in the wireless system. Bio inspired artificial bee colony optimization algorithm is used for evaluating the features of wireless networks. Such algorithms may be incorporated in several business solutions in various competitive technological challenges. This algorithm finds its inspiration in the honey bee colony structure and function. Simple behavior of evaluation and communication seen in the bee agents are implemented in this work. Making asynchronous and decentralized routing decisions are enabled with the help of this algorithm. When compared to the existing nature inspired routing algorithms, the proposed algorithm has several advantages as seen from the outputs of the extensive MATLAB simulations. When simple agent models are used, better performance is achieved using the candidate algorithm. Over large operational areas, the routing protocol and its behavior is studied while evaluating the performance values with the a comprehensive set of values observed in the network. Breadth first search variant is used for deterministically evaluating multiple paths with the candidate algorithm. All possible multiple paths above a certain threshold value is discovered by this algorithm. Improved performance values are achieved by stochastically spreading the data packets on multiple paths using this algorithm. Future work is directed towards discovering multiple paths with multiple threshold values 
Journal of Artificial Intelligence and Capsule Networks (2021)

Vol.03/ No.01

Pages: $62-71$

http://irojournals.com/aicn/

DOI: https://doi.org/10.36548/jaicn.2021.1.006

simultaneously with the routing algorithm while combining the stochastic and deterministic elements.

\section{References}

[1] Varsha, M. B., Kumar, M., \& Kumar, N. (2019). Development of QoS optimized routing using Artificial bee colony and TABU-GA with a mobile base station in Wireless Sensor Network. International Journal of Innovative Technology and Exploring Engineering (IJITEE), 9(1), 926-933.

[2] Famila, S., Jawahar, A., Sariga, A., \& Shankar, K. (2019). Improved artificial bee colony optimization based clustering algorithm for SMART sensor environments. Peer-to-Peer Networking and Applications, 1-9.

[3] Tiwari, V., \& Waoo, A. A. (2020). Comprehensive Study on Metaheuristics FADE Based Artificial Bee Colony Optimization Algorithm to Improve Performance of Wireless Networks.

[4] Al-Ariki, H. D. E., Alareqi, M. A., \& Swamy, S. (2018). An Enhanced Artificial Bee Colony Based EELB-PWDGR for Optimized Route Selection in Wireless Multimedia Sensor Networks. Pertanika Journal of Science \& Technology, 26(4).

[5] Tareq, M., Alsaqour, R., Abdelhaq, M., \& Uddin, M. (2017). Mobile ad hoc network energy cost algorithm based on artificial bee colony. Wireless Communications and Mobile Computing, 2017.

[6] Rambabu, B., Reddy, A. V., \& Janakiraman, S. (2019). Hybrid Artificial Bee Colony and Monarchy Butterfly Optimization Algorithm (HABC-MBOA)-based cluster head selection for WSNs. Journal of King Saud University-Computer and Information Sciences.

[7] Mann, P. S., \& Singh, S. (2017). Artificial bee colony metaheuristic for energy-efficient clustering and routing in wireless sensor networks. Soft Computing, 21(22), 6699-6712.

[8] Vimal, S., Khari, M., Crespo, R. G., Kalaivani, L., Dey, N., \& Kaliappan, M. (2020). Energy enhancement using Multiobjective Ant colony optimization with Double Q learning algorithm for IoT based cognitive radio networks. Computer Communications, $154,481-490$. 
Journal of Artificial Intelligence and Capsule Networks (2021)

Vol.03/ No.01

Pages: $62-71$

http://irojournals.com/aicn/

DOI: https://doi.org/10.36548/jaicn.2021.1.006

[9] Masdari, M., Barshande, S., \& Ozdemir, S. (2019). CDABC: chaotic discrete artificial bee colony algorithm for multi-level clustering in large-scale WSNs. The Journal of Supercomputing, 75(11), 7174-7208.

[10] Mazini, M., Shirazi, B., \& Mahdavi, I. (2019). Anomaly network-based intrusion detection system using a reliable hybrid artificial bee colony and AdaBoost algorithms. Journal of King Saud University-Computer and Information Sciences, 31(4), 541-553.

[11] Raj, J. S. (2020). A Novel Hybrid Secure Routing for Flying Ad-hoc Networks. Journal of trends in Computer Science and Smart technology (TCSST), 2(03), 155-164.

[12] Shakya, S., \& Pulchowk, L. N. (2020). Intelligent and adaptive multi-objective optimization in WANET using bio inspired algorithms. J Soft Comput Paradigm (JSCP), 2(01), 13-23.

[13] Anand, J. V. (2020). Trust-Value Based Wireless Sensor Network Using Compressed Sensing. Journal of Electronics, 2(02), 88-95. 